\title{
Quo Vadis, Molecular Imaging?
}

\author{
Jan Grimm ${ }^{1 *}$, Fabian Kiessling ${ }^{2 *}$, and Bernd J. Pichler ${ }^{3 *}$ \\ ${ }^{I}$ Molecular Pharmacology Program and Department of Radiology, Memorial Sloan Kettering Cancer Center, and Pharmacology \\ Program and Department of Radiology, Weil Cornell Medical College, New York, New York; ${ }^{2}$ Center for Biohybrid Medical Systems, \\ Institute for Experimental Molecular Imaging, University Hospital Aachen, RWTH Aachen University, Aachen, Germany, and \\ Fraunhofer Institute for Digital Medicine, Bremen, Germany; and ${ }^{3}$ Werner Siemens Imaging Center, Department of Preclinical \\ Imaging and Radiopharmacy, Eberhard Karls University, Tuebingen, Germany, and Cluster of Excellence iFIT (EXC 2180) "Image- \\ Guided and Functionally Instructed Tumor Therapies," University of Tübingen, Tübingen, Germany
}

The important insights yielded by molecular imaging (MI) into relevant biologic signatures at an organ-specific and systemic level are not achievable with conventional imaging methods and thus provide an essential link between preclinical and clinical research. New diagnostic probes and imaging methods revealing comprehensive functional and molecular information are being provided by MI research, several of which have found their way into clinical application. However, there are also reservations about the impact of $\mathrm{Ml}$ and its added value over conventional, often less expensive, diagnostic imaging methods. This perspective discusses seminal research directions for the Ml field that have the potential to result in added value to the patient. Emphasis is placed on $\mathrm{MI}$ without probes, $\mathrm{MI}$ based on radiotracers and small molecules, $\mathrm{MI}$ nano- and microsystems, and $\mathrm{MI}$ in context with comprehensive diagnostics. Furthermore, besides technical innovations and probes, emerging clinical indications are highlighted.

Key Words: molecular imaging; diagnostic probe; radiotracer; radiomics; multiparametric imaging

J Nucl Med 2020; 61:1428-1434

DOI: 10.2967/jnumed.120.241984

$\mathbf{M}$ olecular imaging (MI) probes and methodologies often are complex in structure and, because of their high specificity, usually are disease-specific, thus providing advanced differential diagnoses. There is a trend toward multimodal and multiplexed imaging, which requires dedicated readout and image analysis tools, ideally supported by radiomic analysis or artificial intelligence to yield the comprehensive diagnostic power of MI.

The pros and cons of the various MI innovations have to be considered in the context not only of the disease but also of whether a solely diagnostic, theranostic, or image-guided

Received Apr. 27, 2020; revision accepted Aug. 7, 2020.

For correspondence or reprints contact: Jan Grimm, Molecular Pharmacology Program, Memorial Sloan Kettering Cancer Center, 1275 York Ave., New York, NY 10065.

E-mail: grimmj@mskcc.org

${ }^{*}$ Contributed equally to this work.

Published online Aug. 28, 2020.

COPYRIGHT (C) 2020 by the Society of Nuclear Medicine and Molecular Imaging. therapy is ultimately needed. The latter will be of specific interest for cell-based therapies that are on the horizon, such as chimeric antigen receptor $\mathrm{T}$ cells or adoptive $\mathrm{T}$-cell transfers, and provide major opportunities for MI by tailoring therapies according to temporal imaging results. In the context of extremely expensive cellular, immune, or personalized therapies, costs for imaging will be negligible and ultimately will help to reduce total costs by optimizing patient stratification and providing precise diagnostic guidance for personalized therapy.

However, although the imaging-based guidance from cellular therapies can be straightforward when imaging is used for theranostics, there are often discrepancies in dose and toxicity between imaging and therapeutic applications of drugs. A big gap in the realm of theranostics is revealed by the fact that imaging delivers information about pharmacokinetics whereas a therapeutic drug has to be evaluated in the context of pharmacodynamics, toxicity, and its activity profile.

\section{WITHOUT PROBES}

MI with imaging agents provides specific insights into molecular mechanisms, although for some applications, diagnostic findings can also be obtained without specific contrast agents. Naturally, this excludes nuclear medicine approaches, since they cannot sufficiently detect natural isotopes. However, there are modalities capable of providing important endogenous information: optoacoustics, certain optical imaging approaches, and MRI. These modalities, without external probes, are able to provide novel tissuestructural or physiologic-functional information that usually cannot be visualized otherwise.

Ultrasound has already come of age with the emergence of 4-dimensional ultrafast supra-resolution ultrasound, demonstrating unsurpassed anatomic resolution. However, another physical phenomenon has recently opened up ultrasound to true MI without probes: the optoacoustic (or photoacoustic) effect, where pulsed light leads to thermoelastic expansion of chromophores, thus inducing ultrasound signals. There are several intrinsic chromophores in tissues, such as hemoglobin contained within blood vessels. Since oxygenated and deoxygenated hemoglobin can be distinguished, the method 
provides information about tissue oxygenation (1). Another excellent intrinsic absorber is melanin, which has led to clinical application in melanoma surgery to detect positive sentinel lymph nodes in patients (2). Although these studies have been accomplished with lower-resolution scanners, recently a highresolution optoacoustic scanner has been developed and used to characterize the severity of psoriasis in patients (3) and response to antivascular therapy in preclinical models (Fig. 1A) (4). Furthermore, a label-free metabolic imaging method using mid-infrared optoacoustic microscopy was recently introduced, enabling spatiotemporal monitoring of carbohydrates, lipids, and proteins in cells and tissues (Fig. 1B) (5).

Other label-free methods to image endogenous molecules are provided by MRI, such as for assessment of oxygenation and perfusion of tissues by blood oxygenation level-dependent imaging. Furthermore, endogenous molecules can be assessed using chemical-exchange saturation transfer (CEST) (Fig. 1C) (6). CEST is based on the transfer of saturated magnetization to the bulk water signal. In systems with different pools of protons that can be selectively saturated using different radiofrequencies, transfer of saturated protons from one pool to another leads to changes in the signal. For example, gluco-CEST can be used to detect the endogenous glucose concentration in tumors (7). CEST has already found several clinical applications, such as in neurologic diseases and central nervous system tumors (8) and in osteoarthritis (9). Besides this, there is a renaissance of MR spectroscopy, such as for better characterization of human gliomas by metabolic features (Fig. 1C) $(10,11)$.

Molecular tissue characteristics can also be obtained with CT. Recent developments include dual-energy scanners with 2 x-ray sources exhibiting different spectral characteristics to distinguish among calcium, iodine, and soft tissue via differences in absorption characteristics among the various materials, such as for distinguishing small intracranial bleeds from calcium deposits (12). Alternatively, newer technologies enable the detector to discriminate the energy of the x-rays, expanding the application into discrimination of multiple materials and opening avenues for multiparametric imaging (13).

\section{RADIOTRACERS AND SMALL MOLECULES}

Nuclear imaging, specifically PET, not only exhibits the advantage of high detection sensitivity compared with other imaging systems but also offers an exceptional flexibility in the design of radiolabeled probes. Although for many years imaging tracers based on small molecules and peptidessuch as ${ }^{18} \mathrm{~F}$-FDG to quantify glucose metabolism, ${ }^{68} \mathrm{Ga}$-prostatespecific membrane antigen for prostate imaging, or ${ }^{68} \mathrm{Ga}-$ DOTATATE for imaging somatostatin receptors-were used predominantly for biomedical research and clinical diagnosis (14-16), the research focus has shifted toward the latest developments in radiolabeled biologicals, such as antibodies, minibodies, single-domain antibodies, and therapeutic cells. Specifically, the emerging field of immune imaging may open

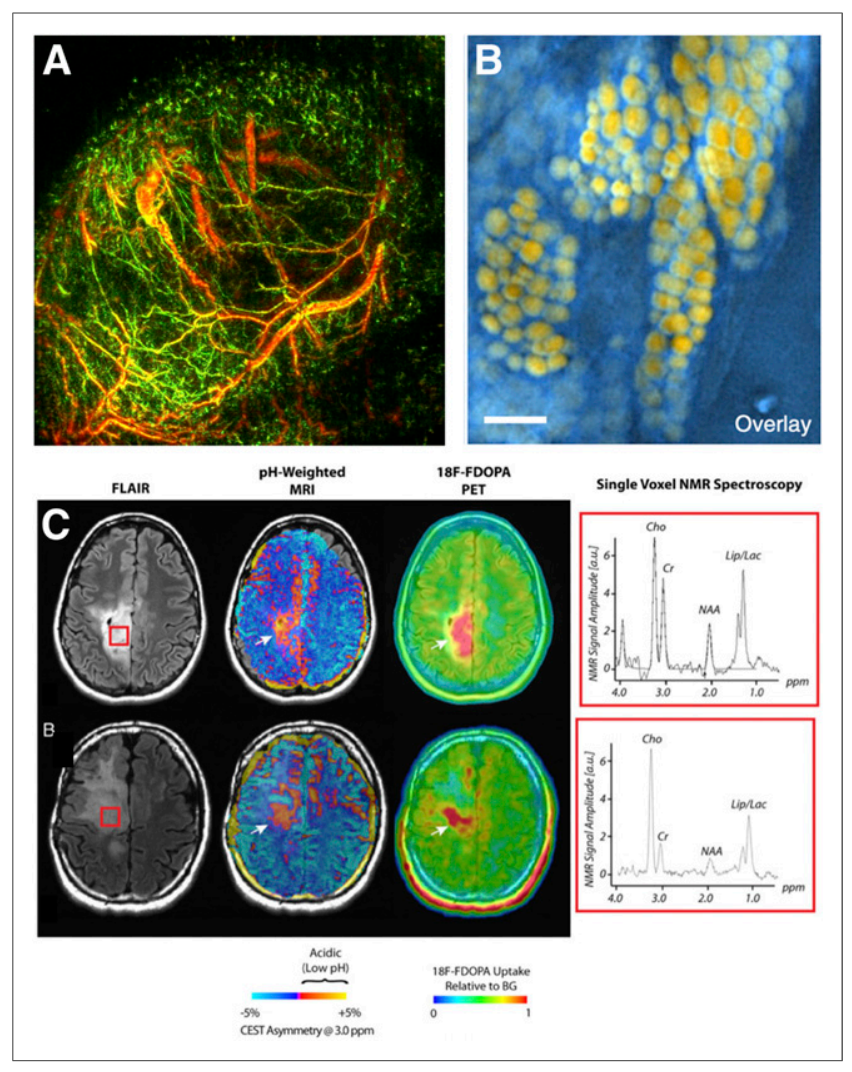

FIGURE 1. Imaging without probes. (A) High-resolution optoacoustic imaging using raster-scanning optoascoustic mesoscopy of subcutaneously implanted CT26 tumor in mouse. (Courtesy of Katja Haedicke and Jan Grimm, Memorial Sloan Kettering Cancer Center.) (B) Mid-infrared optoacoustic microscopy of excised pancreatic mouse tissue, with overlay of lipid $(\mathrm{CH} 2$ [yellow]) and protein (amide [blue]) maps showing clusters of pancreatic acinar glands embedded in protein. (Reprinted with permission of (5).) (C) Spatial correlation between $\mathrm{pH}$-weighted molecular MRI, ${ }^{18} \mathrm{~F}-\mathrm{FDOPA}$ PET, and MR spectroscopy (MRS) in 2 patients with anaplastic astrocytomas showing CEST asymmetry consistent with low $\mathrm{pH}$ in regions with confirmed elevated ${ }^{18} \mathrm{~F}$ FDOPA uptake on PET and elevated lactate on MRS. From left to right are shown T2-weighted fluid-attenuated inversion recovery (FLAIR), pH-weighted MRI using amine CEST, ${ }^{18} \mathrm{~F}$-FDOPA PET, and MRS from area shown in red box in FLAIR images. a.u. = arbitrary units; $\mathrm{BG}=$ background; Cho = choline; $\mathrm{Cr}=$ creatine; FDOPA = 6-fluoro-L-dopa; Lip/Lac = lipids/lactates; NAA = $\mathrm{N}$-acetylaspartate; NMR = nuclear magnetic resonance. (Reprinted with permission of (8).)

unimagined potential in the realm of diagnosis and for imaging-based guidance of therapy in oncology, autoimmune diseases, and neurodegeneration. The outcome of a current clinical study using an ${ }^{89} \mathrm{Zr}$-labeled minibody, specific for CD8 lymphocytes, is eagerly awaited and will potentially show whether immune imaging has the potential to be a paradigm shifter in cancer diagnosis and immunotherapy guidance (17). Other examples are studies with radiolabeled single-domain antibodies to image human epidermal growth factor receptor 2 expression in breast cancer and studies with macrophages $(18,19)$ or biologicals for imaging programmed death 1 or programmed death ligand 1 expression (20). 
The new area of imaging biologicals also poses challenges, and many important details to enable a broad clinical application are not understood yet. The latest technologies offer great flexibility in the design of biologicals but have to compromise among developmental complexity, target specificity, and affinity. At the same time, in vivo pharmacokinetics with physical and biologic half-life have to be optimized. Good clinical manufacturing processes need to be established for a swift clinical translation. All these have to face the complex and usually slow-moving approval processes in multiple countries (Fig. 2). Basic and translational research is needed to unravel for each group of biologicals specific pharmacokinetic properties such as target access in vivo and potential restrictions by size, perfusion, intra- or extracellular target expression, and off-target (or off-tumor) binding. However, full-size antibodies usually allow for straightforward radiolabeling by using chelators and radiometals with a sufficient half-life, matching the relatively slow kinetics and long blood half-life of the antibody. Labeling of smaller biologic agents without altering their shorter blood half-lives and fast compartment-exchange kinetics is usually more complex $(17,21)$ but allows labeling with shorter-lived radioisotopes.

Straightforward conventional but nonspecific radiolabeling approaches are contrasted by more recent developments aiming at a very well defined enzymatic site-specific labeling (22). However, specifically when it comes to clinical translation, the radiolabeling approach — at the end — not only will be a compromise between isotope half-life and radiation dose but also will have to consider simplicity, the required defined

\begin{tabular}{|c|c|c|c|c|c|c|c|c|}
\hline & cells & $\begin{array}{l}\text { Full size } \\
\text { antibodies }\end{array}$ & Minibodies & Diabodies & $\begin{array}{c}\mathrm{Fab} \\
\text { fragments }\end{array}$ & $\begin{array}{c}\mathrm{scFv} \\
\text { tragments }\end{array}$ & Nanobodies & $\begin{array}{c}\text { Small } \\
\text { molecules }\end{array}$ \\
\hline size & $\mu \mathrm{m}$ & $\sim 150 \mathrm{kD}$ & $\sim 80 \mathrm{kD}$ & $\sim 50 \mathrm{kD}$ & $\sim 50 \mathrm{kD}$ & $\sim 25 \mathrm{kD}$ & $\sim 15 \mathrm{kD}$ & $<1 \mathrm{kD}$ \\
\hline $\begin{array}{l}\text { Complexity } \\
\text { develoopment } \\
\& \\
\text { testing }\end{array}$ & $\uparrow$ & $\downarrow$ & $\rightarrow$ & $\rightarrow$ & $\rightarrow$ & $\rightarrow$ & $\rightarrow$ & $\downarrow$ \\
\hline $\begin{array}{l}\text { Biological } \\
\text { activity } \\
z \text { target } \\
\text { specticity }\end{array}$ & $\downarrow / \rightarrow$ & $\uparrow$ & $\rightarrow$ & $\rightarrow$ & $\rightarrow$ & $\rightarrow$ & $\rightarrow$ & $\downarrow / \rightarrow$ \\
\hline $\begin{array}{l}\text { Blood } \\
\text { circulation time } \\
\& \\
\text { required half- } \\
\text { life of isotope }\end{array}$ & (I)/24 & (24) & (I) & () & () & (ా:) & (I: & (ా:/) \\
\hline $\begin{array}{l}\text { Radiolabeling } \\
\text { process } \\
\text { complexity }\end{array}$ & $\rightarrow$ & $\downarrow$ & $\rightarrow / \uparrow$ & $\rightarrow / \uparrow$ & $\rightarrow / \uparrow$ & $\rightarrow / \uparrow$ & $\rightarrow / \uparrow$ & $\downarrow / \rightarrow$ \\
\hline $\begin{array}{l}\text { Isotope } \\
\text { half-life }\end{array}$ & (ए)/24) & (24) & (C)/24 & (ए)/24 & (ए)/24 & (ए)/24 & (ए)/24 & (1:/) \\
\hline $\begin{array}{l}\text { Approval } \\
\text { process } \\
\mathbb{\&} \\
\text { complexity for } \\
\text { clinical } \\
\text { translation }\end{array}$ & $\uparrow$ & $\rightarrow$ & $\rightarrow$ & $\rightarrow$ & $\rightarrow$ & $\rightarrow$ & $\rightarrow$ & $\downarrow$ \\
\hline \multicolumn{9}{|c|}{ (1):Minutes (1) Hours (24)Days } \\
\hline
\end{tabular}

FIGURE 2. MI probes based on biologicals: format defines and impacts not only pharmacokinetics but also development time, costs, and level of effort for clinical translation. Fab fragments $=$ antigen-binding fragments; $\mathrm{scFv}=$ single-chain variable fragment. labeling sites at the biological inflicted by the approval process, and the exclusion of undesired residuals in the labeling process.

Beside radiolabeled small molecules, MRI with hyperpolarized compounds, such as ${ }^{13} \mathrm{C}$ pyruvate, is an emerging field in both preclinical research and the first promising clinical studies (23). Hyperpolarized imaging might synergistically add information to nuclear imaging procedures, enabling a more detailed evaluation of metabolic pathways, specifically in glucose metabolism of tumors.

One development is the emergence of Cerenkov luminescence imaging, a new imaging method using light emitted from decay of radiotracers (24). Cerenkov light arises when charged subatomic particles from radioactive decay travel through tissue faster than the speed of light. It provides several advantages over current optical and nuclear imaging by combining the benefits of optical imaging systems (low camera price, small footprint, high resolution) with the advantages of available PET tracers (high specificity, wide availability). Cerenkov luminescence imaging can tap into the wealth of clinically used radiotracers for optical imaging and has already been translated into the clinic (25).

\section{NANO- AND MICROSYSTEMS}

Nano- and microsystems have frequently been suggested for MI. However, these systems substantially differ in their pharmacokinetic properties from small molecules-a factor that needs to be considered in the conception of molecular diagnostics (Fig. 3): if the size of a nanosystem exceeds that of albumin (5-7 nm), it will not be renally eliminated, and its body retention will be considerably longer. Depending on the system, the result will be a long blood half-life or uptake and persistence in the mononuclear phagocyte system (MPS). Only few systems undergo direct hepatobiliary clearance. The relatively large size of these systems also limits their ability to cross biologic barriers and leads to slow exchange between tissue compartments. On the other hand, in tumors many nanosystems accumulate passively via the enhanced permeability and retention (EPR) effect resulting from the high vascular leakiness and the impairment of lymphatic and venous drainage in tumors. However, these properties of nanosystems make it difficult to design molecular diagnostics, since EPR and MPS uptake generates high nonspecific background. Thus, long intervals $(>6-$ $24 \mathrm{~h}$ ) between probe injection and imaging are often required to depict specific probe binding. 


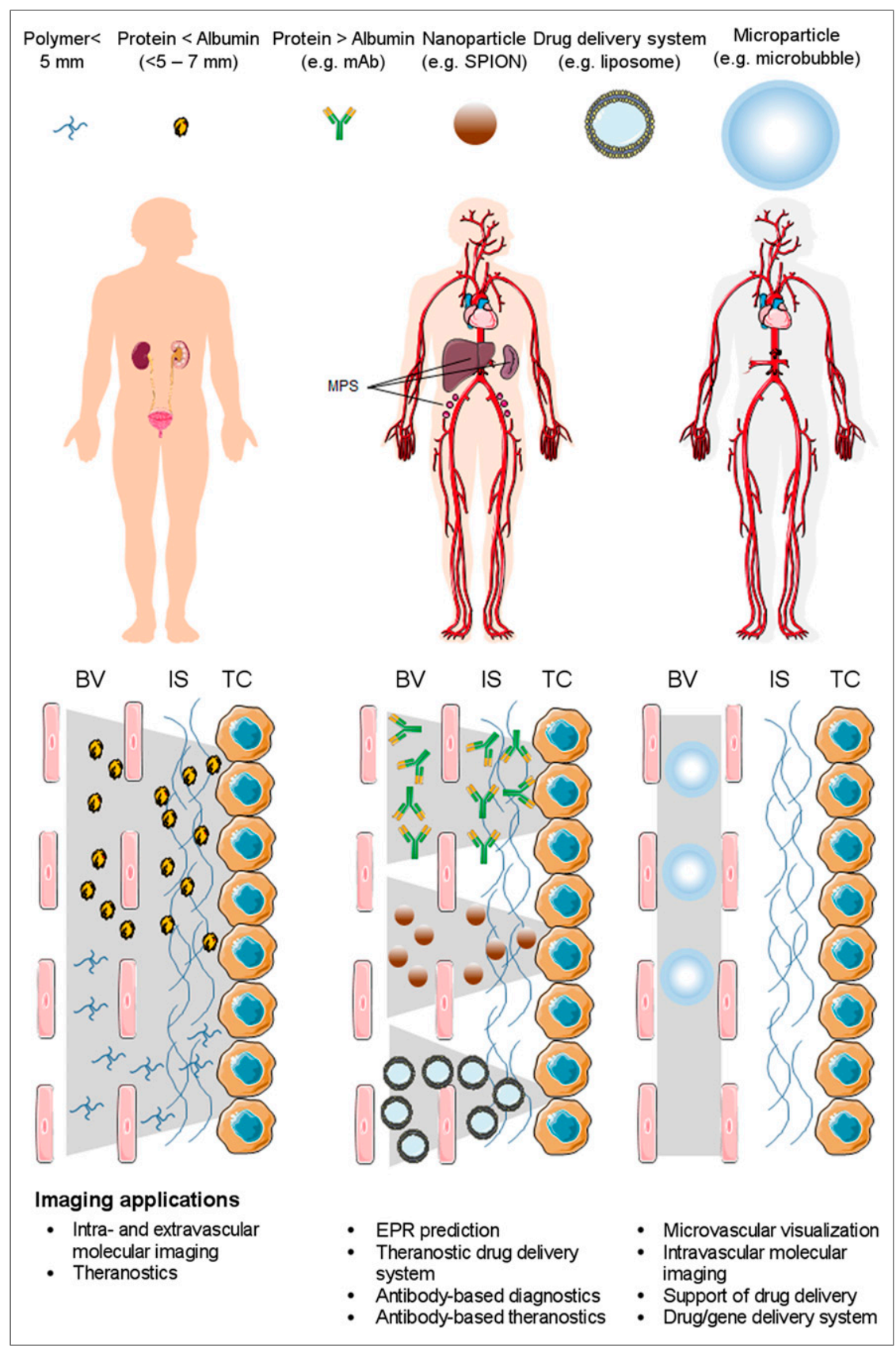

FIGURE 3. Scheme illustrating main sites of accumulation and elimination of diagnostic nanomaterials. This is simplistic view; composition of materials and their interaction with body components may cause different properties in individual cases. Color intensities of human shapes (top row) indicate overall body distribution. (Left) Diagnostic agents smaller than albumin can be renally eliminated and thus usually have short blood half-life and intracorporal persistence. They rapidly overcome biologic barriers, leading to low unspecific accumulation and low background for targeted imaging applications (bottom row). (Middle) Some materials can leave vasculature but are not renally eliminated. They usually show smaller distribution volume but longer body persistence and MPS uptake (liver, spleen, and lymph nodes are shown as an example) and can have long blood half-lives. Diagnostically, these materials can be used for EPR prediction, for MPS staining, and as theranostic drug delivery systems. Antibodies, as considerably small nanosystems ( 12 nm for lgG), also fall into this class. Because of their still-sufficient tissue penetration capabilities, they are frequently used as molecular diagnostic agents, which is reasonable if long intervals between injection and imaging are acceptable in clinical workflow. (Right) Microbubbles can be used as ultrasound contrast agents. Microbubbles remain strictly intravascular and favorably enable vascular visualization and intravascular MI. They are being explored for their capability of locally promoting drug delivery and as carriers of drugs and genes. Gray underlays in bottom row indicate distribution in and penetration of materials into tissue compartments. BV = blood vessel; IS = interstitial space; SPION = superparamagnetic iron oxide nanoparticles; $\mathrm{TC}=$ target cell. (Modified clip art from Servier medical art database [https://smart.servier.com/].)
However, nanomaterials are indicated to assess MPS activity or to predict EPR (26) in tumors. The latter was shown to be highly variable among patients; thus, for many nanotherapies, preselection of patients may be necessary. Ramanathan et al. demonstrated that MRI examinations with superparamagnetic iron oxide nanoparticles can be applied to predict the outcome of a nanoliposomal irinotecan therapy (26). Alternatively, in theranostic applications, nanotherapeutics are directly labeled with imaging probes. The advantage of the latter is a direct feedback on a drug's biodistribution; a disadvantage can be a decrease in a drug's performance due to modification with an imaging tag.

Ultrasound microbubbles are the largest class of MI agents. Ultrasound can detect these probes with high sensitivity and specificity; however, because of their large size, only intravascular targets can be addressed. Currently, a VEGR2-targeted phospholipid-coated system is in clinical evaluation for tumor detection and characterization in breast, ovary, and prostate $(27,28)$. Other systems targeting integrins, selectins, and adhesion molecules are in advanced preclinical evaluation and might be suited for assessing atherosclerosis, vascular injury, and inflammatory bowel disease in patients (27).

In conclusion, small molecules are usually preferable for MI, whereas nanoand microsystems are highly attractive for therapeutic purposes. However, there are exceptions, including radiolabeled or dye-labeled antibodies, agents for assessing MPS, microbubbles for intravascular molecular ultrasound imaging, companion diagnostics (e.g., for EPR), and theranostics.

\section{COMPREHENSIVE DIAGNOSTICS}

Although MI improves diagnosis by assessing one or a few key biomarkers of a certain disease in vivo, comprehensive diagnostics describes the integration of a multitude of diagnostic features in an analytic approach. The analysis of diagnostic features is increasingly supported by artificial intelligence, that is, classic machine learning, deep learning, and neuronal networks (29). If diagnostic features are derived mainly from 
noninvasive images, the term radiomics is used (30); if genomic data are integrated as an additional dimension, we are speaking of radiogenomics. Quantitative imaging data can also be combined with diagnostic information from other sources, such as physical examinations, clinical chemistry, and histopathology (31). Such an integrated diagnostic approach can render diagnoses and prediction of therapeutic outcomes more precise and detailed $(31,32)$. It can also help to elucidate dependencies between various parameters, thereby improving our understanding of pathophysiology and contributing to the evolution of digital disease models. MI can be highly complementary to comprehensive diagnostic approaches since it allows temporal and spatial sampling of molecular marker expression that is not provided by many other diagnostic methods such as histopathology. However, the information provided by MI needs to be nonredundant and meaningful for the diagnostic and therapeutic decision. Unfortunately, the latter is often difficult to predict before the diagnostic power of the clinically available state-of-the-art methods is extensively investigated. This difficulty leads to hesitation by the pharmaceutical industry to invest in new molecular diagnostics.

Nevertheless, it will take time before comprehensive diagnostics show their full potential in the clinic (Fig. 4) $(33,34)$ : training data are often representative of only one site or country, and thus, algorithms show variable performance between sites and are ultimately only as good as the data they are fed. National and international reference datasets are being installed but are not yet available for many diseases. In addition, there are concerns about the protection of patients' privacy when sharing data (34). There are arguments for both keeping reference data in the hospitals and transferring it to the cloud (35). Alternatively, federated learning was suggested, where algorithms from the cloud access local data collections, thereby improving themselves but leaving the data anonymously at the site of origin. As a further option, the use of generative adversarial networks has been proposed to generate artificial datasets from original patient data (36). These artificial datasets can be used as reference data to train decision support systems without touching patients' privacy.

Unfortunately, in many countries digitalization of hospitals is poorly developed and would not allow applying these technologies in clinical routine (35). Furthermore, there is uncertainty about proper approval processes and quality control for artificial-intelligence-based software, particularly if the software continuously learns and modifies its algorithms.

Already, when only exploring the existing diagnostic capabilities, comprehensive diagnostics have the potential to revolutionize diagnostics and clinical workflows and should

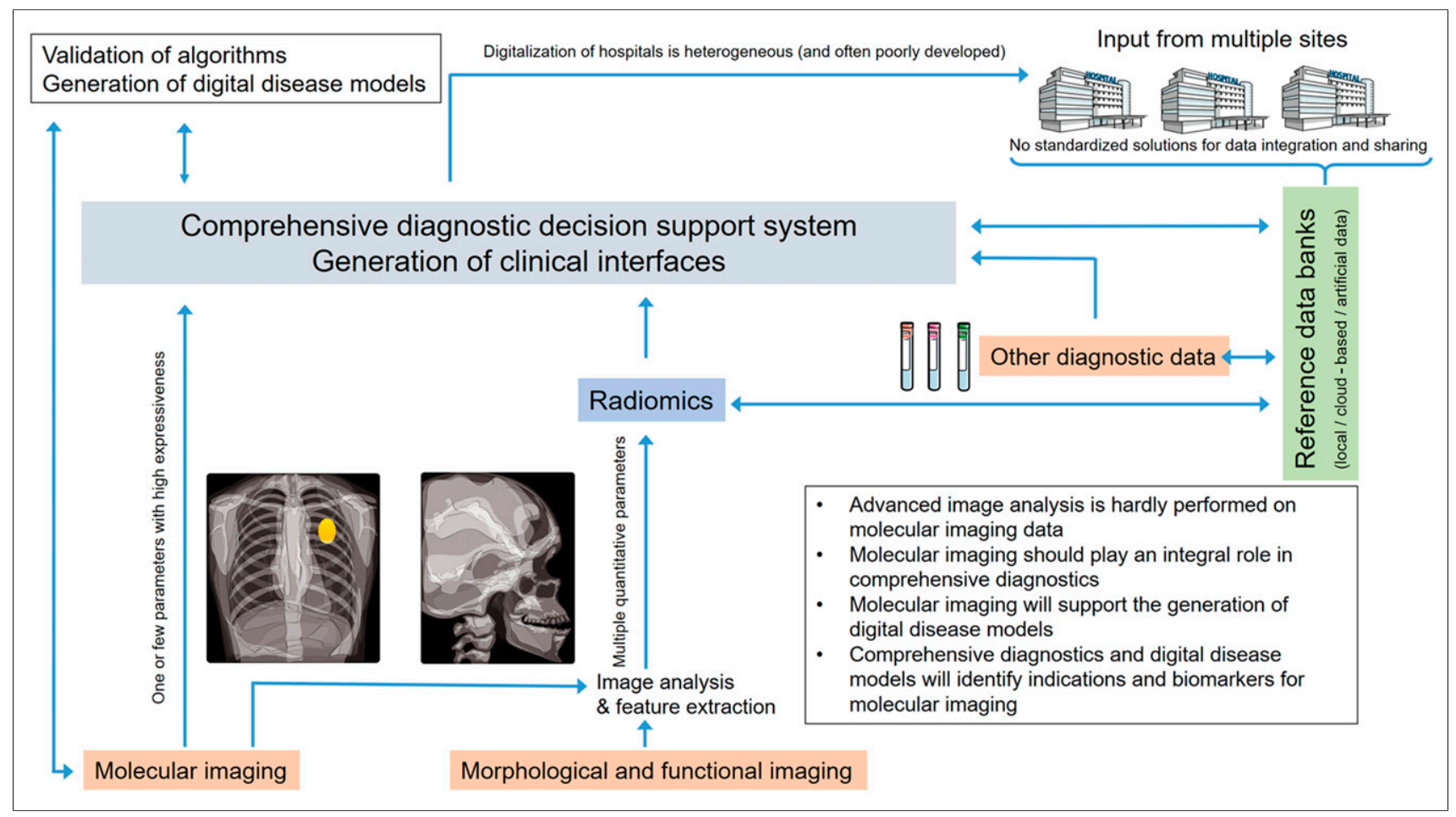

FIGURE 4. Currently, MI aims mostly at extracting one or a few key biomarkers, whereas radiomics aims to achieve diagnostic precision from integrating multiple features extracted from functional and morphologic imaging data. However, multiple features could be extracted from Ml data to enhance its diagnostic accuracy. Integrating imaging features with other diagnostic data (e.g., from physical examinations, electrophysiology, clinical chemistry, and omics) leads to comprehensive diagnostics, where artificial intelligence supports decision making based on learning from large data collections. There is a great need to understand and validate output of artificial intelligence-based decision support systems. Systems biology modeling and generation of virtual disease models will become important. These models will profit from Ml of key parameters of pathologic processes and inversely provide indications for developing new Ml probes. (Modified clip art from Servier medical art database [https://smart.servier.com/].) 
be considered when deciding on the need to develop new MI probes. MI should provide access to specific key biomarkers that are lacking in diagnostic assays and that are key for understanding the interplay of diagnostic features and, thus, of pathophysiology.

\section{NEW INDICATIONS FOR MI}

In the available space for this article, it is impossible to comprehensively review new indications. Only a few examples will therefore be highlighted. Most prominently, MI is playing an increasing role in selecting patients for targeted therapies. For example, in immunotherapy, MI can determine which patients will benefit from the expensive intervention (37). Furthermore, with the emergence of infectious diseases as critical threads in global public health and economies, imaging of infection will assuredly receive more prominence in future applications (38). New and expanded modalities such as the above-mentioned supra-resolution ultrasound and optoacoustic methods will transit into the clinic to provide unprecedented information, possibly together with repurposed or new agents. Another aspect of MI that is probably important is its expansion into the field of in vitro diagnostics, such as in the form of matrix-assisted laser desorption/ionization time-of-flight imaging (39) and imaging-based in vitro assays (Fig. 5). A recent example is a continuously infused microfluidic radioassay with a miniaturized PET scanner for microfluidics-based radiobioassays with a wide range of applications such as evaluations of organoids, clinical samples, and lab-on-a-chip studies (40).

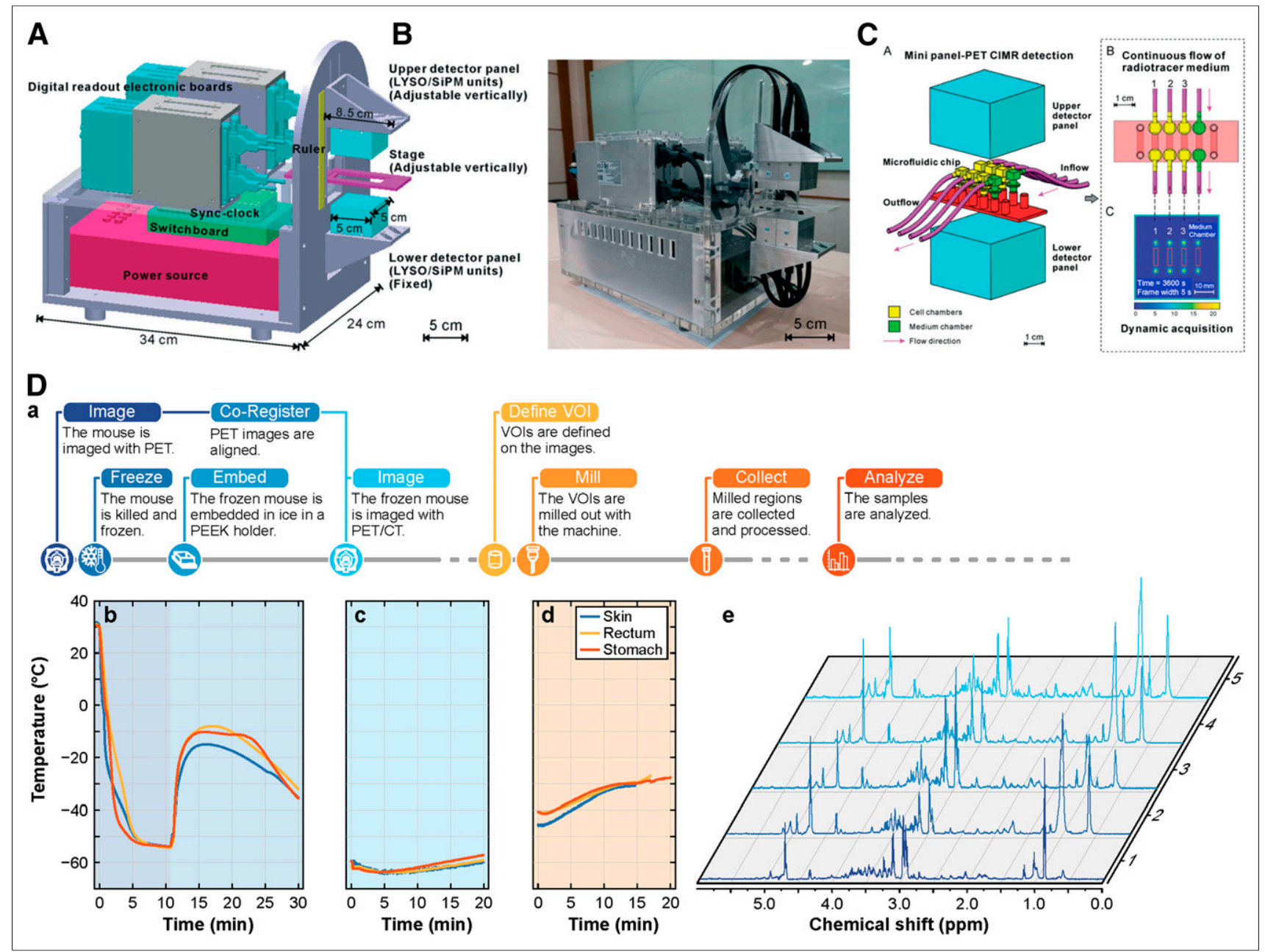

FIGURE 5. (A) Three-dimensional computer-aided design sketch of modularized mini-panel PET detection system. (B) Photograph of system. (C) Core setup of microfluidics system (a), continuous flow of radiotracer medium (b), and representative image from PET detector during acquisition (c). Red boxes denote analyzed areas (40). (D) Example of comprehensive data integration that connects workflows of in vivo $\mathrm{Ml}$ and in vitro postmortem tissue metabolomics. (a) Flowchart of procedure for image-guided tissue extraction. (b-d) Temperature measured with sensors in stomach, in rectum, and embedded under skin during freezing and embedding (b), during time that mouse was kept at room temperature and covered with dry ice (c), and during milling (d). (e) Example of nuclear magnetic resonance spectra from samples obtained with image-guided milling machine. CIMR = continuously infused microfluidic radioassay; LYSO/SiPM = lutetium yttrium oxyorthosilicate/silicon photomultiplier; PEEK = polyether ether ketone; $\mathrm{VOI}=$ volume of interest. (Reprinted with permission of (43).) 


\section{OUTLOOK}

The latest developments in multimodal imaging modalities $(41,42)$ and novel imaging tracers, based on either small molecules or biologicals, clearly indicate that the MI field grasps the diagnostic approaches needed toward highly complex multifactorial diseases such as cancer and neurodegeneration. Imaging-based guidance is needed beyond that available from standard morphologic tomographic imaging or ${ }^{18} \mathrm{~F}$-FDG PET to carve out the strength of comprehensive imaging information and compete with in vitro diagnostics. Thus, convergence of different imaging modalities and imaging probes is urgently required, along with advanced image analysis exploiting the potential of artificial intelligence and machine learning for multiparametric image analysis. We are just at the threshold of an exciting time for MI, paving the road for affordable personalized medicine. Thus, regarding MI, we do not ask quo vadis - where is it goingin the sense used by the baroque artist Annibale Carracci, whose painting "Domine, Quo Vadis?" depicts the resurrected Jesus carrying his cross to Rome to be crucified a second time. Rather, we ask quo vadis as the important question of finding the right strategy for placing this powerful imaging discipline in a new era of diagnostics for a new resurrection.

\section{DISCLOSURE}

No potential conflict of interest relevant to this article was reported.

\section{REFERENCES}

1. Knieling F, Neufert C, Hartmann A, et al. Multispectral optoacoustic tomography for assessment of Crohn's disease activity. N Engl J Med. 2017;376:1292-1294.

2. Stoffels I, Morscher S, Helfrich I, et al. Metastatic status of sentinel lymph nodes in melanoma determined noninvasively with multispectral optoacoustic imaging. Sci Transl Med. 2015;7:317ra199.

3. Schwarz M, Garzorz-Stark N, Eyerich K, Aguirre J, Ntziachristos V. Motion correction in optoacoustic mesoscopy. Sci Rep. 2017;7:10386.

4. Haedicke K, Agemy L, Omar M, et al. High-resolution optoacoustic imaging of tissue responses to vascular-targeted therapies. Nat Biomed Eng. 2020;4:286-297.

5. Pleitez MA, Khan AA, Solda A, et al. Label-free metabolic imaging by mid-infrared optoacoustic microscopy in living cells. Nat Biotechnol. 2020;38:293-296.

6. Goergen CJ, Sosnovik DE. From molecules to myofibers: multiscale imaging of the myocardium. J Cardiovasc Transl Res. 2011;4:493-503.

7. Chan KW, McMahon MT, Kato Y, et al. Natural D-glucose as a biodegradable MRI contrast agent for detecting cancer. Magn Reson Med. 2012;68:1764-1773.

8. Harris RJ, Cloughesy TF, Liau LM, et al. pH-weighted molecular imaging of gliomas using amine chemical exchange saturation transfer MRI. Neuro Oncol. 2015; 17:1514-1524

9. Jones KM, Pollard AC, Pagel MD. Clinical applications of chemical exchange saturation transfer (CEST) MRI. J Magn Reson Imaging. 2018;47:11-27.

10. Choi C, Ganji SK, DeBerardinis RJ, et al. 2-hydroxyglutarate detection by magnetic resonance spectroscopy in IDH-mutated patients with gliomas. Nat Med. 2012;18: $624-629$.

11. de la Fuente MI, Young RJ, Rubel J, et al. Integration of 2-hydroxyglutarate-proton magnetic resonance spectroscopy into clinical practice for disease monitoring in isocitrate dehydrogenase-mutant glioma. Neuro Oncol. 2016;18:283-290.

12. Wiggins WF, Potter CA, Sodickson AD. Dual-energy CT to differentiate small foci of intracranial hemorrhage from calcium. Radiology. 2020;294:129-138.

13. Fornaro J, Leschka S, Hibbeln D, et al. Dual- and multi-energy CT: approach to functional imaging. Insights Imaging. 2011;2:149-159.

14. Pinker K, Riedl C, Weber WA. Evaluating tumor response with FDG PET: updates on PERCIST, comparison with EORTC criteria and clues to future developments. Eur J Nucl Med Mol Imaging. 2017;44:55-66.
15. Schwenck J, Rempp H, Reischl G, et al. Comparison of ${ }^{68} \mathrm{Ga}$-labelled PSMA-11 and ${ }^{11} \mathrm{C}$-choline in the detection of prostate cancer metastases by PET/CT. Eur J Nucl Med Mol Imaging. 2017;44:92-101.

16. Mikołajczak R, Maecke HR. Radiopharmaceuticals for somatostatin receptor imaging. Nucl Med Rev Cent East Eur. 2016;19:126-132.

17. Pandit-Taskar N, Postow MA, Hellmann MD, et al. First-in-humans imaging with ${ }^{89} \mathrm{Zr}$-Df-IAB22M2C anti-CD8 minibody in patients with solid malignancies: preliminary pharmacokinetics, biodistribution, and lesion targeting. $\mathrm{J} \mathrm{Nucl} \mathrm{Med}$. 2020;61:512-519.

18. Keyaerts M, Xavier C, Heemskerk J, et al. Phase I study of ${ }^{68} \mathrm{Ga}-\mathrm{HER} 2$-nanobody for PET/CT assessment of HER2 expression in breast carcinoma. J Nucl Med. 2016;57:27-33.

19. Xavier C, Blykers A, Laoui D, et al. Clinical translation of $\left[{ }^{68} \mathrm{Ga}\right] \mathrm{Ga}-\mathrm{NOTA}$-antiMMR-sdAb for PET/CT imaging of protumorigenic macrophages. Mol Imaging Biol. 2019;21:898-906.

20. Niemeijer AN, Leung D, Huisman MC, et al. Whole body PD-1 and PD-L1 positron emission tomography in patients with non-small-cell lung cancer. Nat Commun. 2018;9:4664.

21. Debie P, Lafont C, Defrise M, et al. Size and affinity kinetics of nanobodies influence targeting and penetration of solid tumours. J Control Release. 2020;317:34-42.

22. Pishesha N, Ingram JR, Ploegh HL, Sortase A. A model for transpeptidation and its biological applications. Annu Rev Cell Dev Biol. 2018;34:163-188.

23. Gallagher FA, Woitek R, McLean MA, et al. Imaging breast cancer using hyperpolarized carbon-13 MRI. Proc Natl Acad Sci U S A. 2020;117:2092-2098.

24. Ruggiero A, Holland JP, Lewis JS, Grimm J. Cerenkov luminescence imaging of medical isotopes. J Nucl Med. 2010;51:1123-1130.

25. Thorek DL, Riedl CC, Grimm J. Clinical Cerenkov luminescence imaging of ${ }^{18}$ F-FDG. J Nucl Med. 2014;55:95-98.

26. Ramanathan RK, Korn RL, Raghunand N, et al. Correlation between ferumoxytol uptake in tumor lesions by MRI and response to nanoliposomal irinotecan in patients with advanced solid tumors: a pilot study. Clin Cancer Res. 2017;23:3638-3648.

27. Güvener N, Appold L, de Lorenzi F, et al. Recent advances in ultrasound-based diagnosis and therapy with micro- and nanometer-sized formulations. Methods. 2017;130:4-13.

28. Zlitni A, Gambhir SS. Molecular imaging agents for ultrasound. Curr Opin Chem Biol. 2018;45:113-120.

29. Esteva A, Robicquet A, Ramsundar B, et al. A guide to deep learning in healthcare. Nat Med. 2019;25:24-29.

30. Dercle L, Lu L, Schwartz LH, et al. Radiomics response signature for identification of metastatic colorectal cancer sensitive to therapies targeting EGFR pathway. J Natl Cancer Inst. February 4, 2020 [Epub ahead of print].

31. Papp L, Potsch N, Grahovac M, et al. Glioma survival prediction with combined analysis of in vivo ${ }^{11} \mathrm{C}$-MET PET features, ex vivo features, and patient features by supervised machine learning. J Nucl Med. 2018;59:892-899.

32. Dissaux G, Visvikis D, Da-Ano R, et al. Pretreatment ${ }^{18}$ F-FDG PET/CT radiomics predict local recurrence in patients treated with stereotactic body radiotherapy for early-stage non-small cell lung cancer: a multicentric study. J Nucl Med. 2020;61:814-820.

33. Lambin P, Leijenaar RTH, Deist TM, et al. Radiomics: the bridge between medical imaging and personalized medicine. Nat Rev Clin Oncol. 2017;14:749-762.

34. Topol EJ. High-performance medicine: the convergence of human and artificial intelligence. Nat Med. 2019;25:44-56.

35. Bukowski M, Farkas R, Beyan O, et al. On the way to digital comprehensive diagnostics: a comparative analysis of current international activities. Eur Radiol. In press

36. Han T, Nebelung S, Haarburger C, et al. Breaking medical data sharing boundaries by employing artificial radiographs. BioRxiv website. https://www.biorxiv.org/content/ 10.1101/841619v1. Published November 14, 2019. Accessed July 27, 2020.

37. van de Donk PP, Kist de Ruijter L, Lub-de Hooge MN, et al. Molecular imaging biomarkers for immune checkpoint inhibitor therapy. Theranostics. 2020;10:1708-1718.

38. Ordonez AA, Wang H, Magombedze G, et al. Dynamic imaging in patients with tuberculosis reveals heterogeneous drug exposures in pulmonary lesions. Nat Med. 2020;26:529-534.

39. Kriegsmann J, Kriegsmann M, Casadonte R. MALDI TOF imaging mass spectrometry in clinical pathology: a valuable tool for cancer diagnostics. Int $J$ Oncol. 2015;46:893-906.

40. Liu Z, Zhang P, Ji H, et al. A mini-panel PET scanner-based microfluidic radiobioassay system allowing high-throughput imaging of real-time cellular pharmacokinetics. Lab Chip. 2020;20:1110-1123.

41. Mannheim JG, Schmid AM, Schwenck J, et al. PET/MRI hybrid systems. Semin Nucl Med. 2018;48:332-347.

42. Provost J, Garofalakis A, Sourdon J, et al. Simultaneous positron emission tomography and ultrafast ultrasound for hybrid molecular, anatomical and functional imaging. Nat Biomed Eng. 2018;2:85-94.

43. Disselhorst JA, Krueger MA, Ud-Dean SMM, et al. Linking imaging to omics utilizing image-guided tissue extraction. Proc Natl Acad Sci USA. 2018;115:E2980-E2987. 